\title{
Understanding Long-Term Cancer Survivors' Preferences for Ongoing Medical Care
}

\author{
Tenbroeck G. Smith, $\mathrm{MA}^{7}$, Sara Strollo, $\mathrm{MPH}^{7}$, Xin $H u, M S P H^{2}$, Craig C. Earle, $\mathrm{MD}^{3}$, \\ Corinne R. Leach, $\mathrm{PhD}^{7}$, and Larissa Nekhlyudov, MD, $\mathrm{MPH}^{4}$
}

'Behavioral and Epidemiology Research Group, American Cancer Society, Atlanta, GA, USA; ${ }^{2}$ Yale Center for Analytical Sciences, Yale School of Public Health, New Haven, CT, USA; ${ }^{3}$ Ontario Institute for Cancer Research, Toronto, Canada; 'Department of Medicine, Brigham \& Women's Hospital, Boston, MA, USA.

BACKGROUND: Due to risk for treatment-related late effects and concerns about cancer recurrence, long-term cancer survivors have unique medical needs. Survivors' preferences for care may influence adherence and care utilization.

OBJECTIVE: To describe survivors' preferences for care and factors associated with preferred and actual care.

DESIGN: Cross-sectional analysis of participants in a longitudinal study using mailed questionnaires.

PARTICIPANTS: Survivors of ten common cancers ( $n=$ 2,107 , mean years from diagnosis 8.9).

MAIN MEASURES: (1) Survivors' preferences for primary care physician (PCP) and oncologist responsibilities across four types of care: cancer follow-up, cancer screening, preventive health, and comorbid conditions. (2) Survivor-reported visits to PCPs and oncologists.

KEY RESULTS: The response rate was 42.1\%. Most longterm survivors preferred PCPs and oncologists share care for cancer follow-up (63\%) and subsequent screening (65\%), while preferring PCP-led preventive health $(77 \%)$ and comorbid condition (83\%) care. Most survivors (88\%) preferred oncologists involved in cancer follow-up care, but only $60 \%$ reported an oncologist visit in the previous 4 years, and 96\% reported a PCP visit in the previous 4 years. In multivariable regressions, those with higher fear of cancer recurrence were less likely to prefer PCP-led cancer follow-up care $(\mathrm{OR}=0.96, \mathrm{CI}=0.93-0.98)$, as did survivors with advanced cancer stage $(\mathrm{OR}=0.56, \mathrm{CI}=$ $0.39-0.79)$. Those with higher fear of recurrence $(\mathrm{OR}=$ $1.03, \mathrm{CI}=1.01-1.04$ ) or who preferred oncologist-led cancer follow-up care $(\mathrm{OR}=2.08, \mathrm{CI}=1.63-2.65)$ had greater odds of seeing an oncologist in the last 4 years.

CONCLUSIONS: Most cancer survivors preferred PCPs and oncologists share care for cancer follow-up and screening, yet many had not seen an oncologist recently. Survivors preferred PCP-led care for other preventive services and management of comorbid conditions. These findings highlight the important role PCPs could play in survivor care, suggesting the need for PCP-oriented

$\overline{\overline{P r i o r} \text { Presentations These analyses have not been previously presented. }}$ Electronic supplementary material The online version of this article (https://doi.org/10.1007/s11606-019-05189-y) contains supplementary material, which is available to authorized users.

Received August 14, 2018

Revised February 21, 2019

Accepted June 21, 2019

Published online July 31, 2019 education and health system policies that support highquality PCP-led survivor care.

KEY WORDS: cancer survivorship; primary care; patient-centered care; oncology; fear of cancer recurrence.

J Gen Intern Med 34(10):2091-7

DOI: $10.1007 / \mathrm{s} 11606-019-05189-\mathrm{y}$

() Society of General Internal Medicine 2019

\section{INTRODUCTION}

There were over 15.5 million cancer survivors in the USA in 2016 , and that number is expected to grow to 20 million by 2026 due to increasing numbers of older Americans as well as improvements in early detection and treatment. ${ }^{1}$ After the completion of curative treatment, cancer survivors face numerous long-term health risks due to the effects of cancer and its treatment. ${ }^{2,3}$ Survivors' original cancer may recur, and they are at higher risk than the general public for new, biologically distinct cancers. ${ }^{4}$ Cancer-related symptoms, such as fatigue, distress, or fear of recurrence, may begin around the time of diagnosis and persist for years. Physical late effects, such as cardiotoxicity, ${ }^{5}$ may not emerge until years after completion of treatment. Therefore, quality and timely cancer survivorship care is crucial and may be organized into four categories: (1) cancer follow-up care including surveillance for recurrence and management of side effects; (2) screening for new, biologically distinct cancers; (3) preventive health services; and (4) care for comorbid conditions. 6, 7

It is not clear which clinicians - oncologists or primary care physicians (PCPs) - are better suited to provide survivor care. Oncologists are generally confident in their knowledge of cancer-related aspects of survivor care (e.g., cancer recurrence, physical late effects), and expect to play a leading role in these areas. ${ }^{8-10}$ In contrast, many PCPs express concerns about their knowledge about cancer recurrence and late effects. ${ }^{10,11} \mathrm{On}-$ cologists were more likely than PCPs to correctly identify late effects caused by specific chemotherapeutic agents. ${ }^{12}$ These attitudes are reflected in lower rates of surveillance for recurrence among survivors seen by PCPs only. ${ }^{13-15}$ On the other hand, oncologists expect to play less of a role in providing other aspects of survivor care such as screening for other 
cancers, preventive services, and management of comorbid conditions. ${ }^{8,16}$ Survivors seen exclusively by oncologists receive less optimal preventive care (e.g., influenza vaccine) than those seen by PCPs ${ }^{17}$ and PCPs play a critical role in the quality of care for comorbid conditions. ${ }^{18,19}$ These patterns suggest that shared oncologist-PCP care may facilitate more comprehensive survivorship care. ${ }^{20}$ However, shared care requires coordination among clinicians ${ }^{21}$ and may result in overutilization and higher costs, known issues in survivor care. $^{22-24}$

Patient-centered care emphasizes the importance of patientclinician communication, shared decision making, and respect for patient preferences. ${ }^{25}$ Patient-centered care aligns with the core principles of medical ethics ${ }^{26}$ and has been associated with improvements in patient satisfaction, adherence, and selfmanagement ${ }^{27}$. Understanding survivors' preferences can help clinicians deliver patient-centered care.

In this paper, we describe long-term survivors' preferences for receiving care from PCP or oncologists for four types of care (cancer follow-up care, screening for new cancers, preventive health, and comorbid condition care). We investigate the factors that influence those preferences and how those preferences are related to survivors' actual provider visits.

\section{METHODS}

\section{Study Design}

We report data from the third wave of the American Cancer Society's Study of Cancer Survivors-I (SCS-I), a longitudinal, nationwide study of adult cancer survivors' quality of life and adjustment. SCS-I was approved by Emory University IRB with rationale and methodology detailed elsewhere. ${ }^{28}$ SCS-I included adult, English-/Spanish-speaking people diagnosed with one of 10 common cancers (breast, prostate, bladder, uterine, melanoma, colorectal, kidney, non-Hodgkin lymphoma, ovarian, and lung) from January 2001 to September 2003. Population-based samples were drawn from 11 state cancer registries (AL, CT, IA, ID, MN, NJ, OH, PA, RI, SC, and WY), stratified by state, age, cancer type, and race/ethnicity. Survivors younger than 55 years and racial and ethnic minorities were oversampled. Recruitment via mixed mail and telephone yielded a $93.0 \%$ physician consent rate and a $42.1 \%$ case response rate, ${ }^{28}$ resulting in 6309 participants; of those, $49.7 \%(n=3,138)$ completed the third-wave questionnaire that contained our outcomes. In order to focus on diseasefree survivors, we excluded those reporting progression of their original cancer diagnosis or a new cancer diagnosis in the past 5 years $(n=422)$. Other exclusions included those missing data on care preferences $(n=354)$, oncologist $(n=$ $137)$ or PCP $(n=33)$ visits, or any covariates $(n=85)$, resulting in an analytic sample of 2107 . To explore missing data bias, we compared the analytic sample to those individuals excluded due to missing data (see Online Appendix Table 1). Most differences between these groups had negligible to weak effect sizes. While older individuals were more likely to provide incomplete data, the mean difference in age between the groups was only 5 years.

\section{Covariate Measures}

Sociodemographic variables and cancer events in the last 5 years were self-reported, and cancer type and stage came from the cancer registries. Because certain cancer types are not independent of sex, we created a six-category variable: breast (females only); prostate; colorectal female; colorectal male; other female; and other male. The other categories include survivors of non-Hodgkin lymphoma, melanoma, lung, uterine, kidney, ovarian, or urinary bladder cancers.

Fear of cancer recurrence is common among cancer survivors and influences health behaviors, care satisfaction, and healthcare utilization, ${ }^{29,30}$ and may influence care preferences. Fear of recurrence can be assessed and reduced ${ }^{29}$; thus, it may be a modifiable influencer of preferences. Fear of recurrence is measured with the severity subscale of the Fear of Cancer Recurrence Inventory (FCRI), a validated instrument. ${ }^{31}$ The severity subscale contains nine questions assessing respondents' perceived risk of recurrence, fear of recurrence, and frequency of thinking about recurrence; responses are provided on a 5-point scale ranging from "not at all" to "a great deal." The items are summed to create a continuous scale score (range $=0$ 36 , Cronbach's alpha $=.85$ ).

\section{Outcome Measures}

Survivors' preferences for four types of care were assessed with previously developed questions. ${ }^{8}$ For each type of care, survivors were asked two similar questions: "In your opinion, how much responsibility should each of these doctors (1) my cancer doctor or (2) my primary care doctor have to...": (1) "follow you up for your most recent cancer," (2) "screen you for cancers other than your most recent cancer," (3) "provide general preventive health care, like flu shots, cholesterol testing, etc.," and (4) "treat other medical problems besides cancer, such as diabetes, high blood pressure, arthritis, etc." (i.e., comorbid conditions). Response options were "None," "A little," "Some," "A lot," or "Full." Each survivor's responses were combined to characterize their preferences for each type of care as oncologist-led, shared, or PCP-led. Survivors who rated oncologist's responsibility at least two levels higher than PCP's responsibility were categorized as preferring oncologist-led; those rating oncologist/PCP responsibilities within two levels were categorized as shared; and those rating PCP's responsibility at least two levels higher than oncologist's were categorized as PCP-led (see Online Appendix Tables 2-5).

Survivors' actual care was assessed by asking, "How long has it been since you received health care from the following providers?": (1) "A cancer doctor, such as a medical oncologist, surgical oncologist, or radiation oncologist."; (2) "A primary care doctor, such as a family doctor, general internist, 
or non-cancer specialist. You may have been seeing your primary care doctor for years or they may have been assigned to you by your insurance company or health plan." Response options were "Less than 1 year ago," "1-2 years ago", "3-4 years ago," or "5 or more years ago." Oncologist visits were dichotomized to indicate those who had seen an oncologist in the last 4 years. Since $96 \%$ of survivors had seen a PCP in the last 4 years, this variable was not analyzed further.

\section{Statistical Analysis}

Descriptive analyses were performed to summarize sociodemographic and clinical characteristics, preferences for each aspect of care, and time since most recent care. A multinomial logistic regression explored associations between individual characteristics (age, race/ethnicity, marital status, education level, health insurance status, cancer type by sex, cancer stage, and fear of cancer recurrence severity) and preference for follow-up cancer care (oncologist-led, shared, or PCP-led). A logistic regression revealed associations between individual characteristics, survivor's preferences for care, and use of an oncologist in the last 4 years. All analyses used SAS version 9.4 (SAS Institute Inc. Cary, NC).

\section{RESULTS}

Sociodemographic and clinical characteristics of the analytic sample of disease-free cancer survivors are highlighted in Table $1(n=2107)$. Participants were a mean of 8.9 years from diagnosis (standard deviation $(\mathrm{SD})=0.7$; range $7.4-11.3$ ).

Table 1 Individual and Cancer-Related Characteristics $(N=2107)$

\begin{tabular}{ll}
\hline \hline Characteristics & $\boldsymbol{N}(\boldsymbol{\%})$ \\
\hline Mean age, years (SD) & $64.5(11.4)$ \\
Race/ethnicity & $1901(90.2)$ \\
Non-Hispanic white & $206(9.8)$ \\
Other & $1551(73.6)$ \\
Marital status & $431(20.5)$ \\
Married/cohabitating & $125(5.9)$ \\
Separated/divorced/widowed & \\
Single & $659(31.3)$ \\
Education & $1448(68.7)$ \\
High school or less & \\
More than high school & $2052(97.4)$ \\
Health insurance & $55(2.6)$ \\
Yes & \\
No & $652(30.9)$ \\
Cancer type by gender & $424(20.1)$ \\
Breast & $115(5.5)$ \\
Prostate & $170(8.1)$ \\
Colorectal male & $255(12.1)$ \\
Colorectal female & $491(23.3)$ \\
Other male & \\
Other female & $1470(69.8)$ \\
Cancer stage & $637(30.2)$ \\
In situ/localized & \\
Regional/distant & $1261(59.8)$ \\
Oncologist visit within 4 years & $846(40.2)$ \\
Yes & $11.0(6.9)$ \\
No & $8.9(0.7)$ \\
Mean fear of cancer recurrence (SD) &
\end{tabular}

$S D$, standard deviation
Breast and prostate cancers were the most common diagnoses. The mean age was 64.5 years $(\mathrm{SD}=11.4)$. Most of the sample were female (62\%), non-Hispanic white (90\%), and married or cohabitating (74\%). Most participants received education higher than the high school level (69\%) and reported health insurance coverage (97\%). Fear of recurrence was elevated in $39 \%(n=822)$ of the sample, whose FCRI score was at or above the clinical screening cut point (FCRI $\geq 13){ }^{32}$

Most respondents preferred cancer follow-up care be shared between oncologist and PCP (63\%), though some preferred oncologist-led (25\%) and PCP-led (12\%) care (Fig. 1). When screening for cancers other than the one with which they were diagnosed, most respondents preferred a shared model $(65 \%)$, with $21 \%$ preferring PCPled and $14 \%$ oncologist-led. PCP-led care was mostly preferred for preventative health $(77 \%)$ and comorbid conditions $(83 \%)$. Among participants with data on all four types of care ( $n=1849)$ - cancer follow-up, cancer screening, preventive health, comorbid conditions - most survivors preferred a mixture of models $(79 \%, n=1464)$, some exclusively preferred shared $(12 \% ; n=216)$ or PCP-led care $(9 \% ; n=163)$, and very few $(<1 \% ; n=6)$ survivors wanted solely oncologist-led care. Most survivors $(91 \%, n=1680)$ wanted both types of providers involved in their care.

Table 2 shows the multivariable, multinomial logistic model of predictors of care preferences using shared care as the reference. Those with higher education level (odds ratio $[\mathrm{OR}]=1.50,95 \%$ confidence interval $[\mathrm{CI}]=1.19-1.89)$ and those with health insurance $(\mathrm{OR}=3.46, \mathrm{CI}=1.35-8.89)$ were more likely to prefer oncologist-led to shared care. Compared with female breast cancer survivors, prostate cancer survivors were less likely to prefer oncologist-led care $(\mathrm{OR}=0.69, \mathrm{CI}=$ 0.51-0.95) to shared care. Switching to the comparison of PCP-led with shared care, prostate $(\mathrm{OR}=1.67, \mathrm{CI}=1.11$ $2.50)$, colorectal female $(\mathrm{OR}=1.90, \mathrm{CI}=1.10-3.26)$, and other female $(\mathrm{OR}=1.50, \mathrm{CI}=1.00-2.25)$ survivors were more likely to prefer PCP-led to shared care versus breast cancer survivors. Compared with survivors with localized cancer stages, regional/distant stage survivors had $44 \%(\mathrm{CI}=21 \%$ $61 \%$ ) lower odds of preferring PCP-led to a shared care. Higher fear of recurrence scores were associated with lower odds of preferring PCP-led care to shared care $(\mathrm{OR}=0.96, \mathrm{CI}$ 0.93-0.98 for 1 point; $\mathrm{OR}=0.73$, CI $0.63-0.86$ for 1 standard deviation (6.9)). These results reflect that survivors of breast cancer, those with regional/distant stage of disease, and those with higher levels of fear of recurrence preferred an oncologist involved in their care via either oncologist-led or shared care.

Among these long-term cancer survivors, $60 \%$ reported visiting an oncologist and $96 \%$ a PCP within the past 4 years. Further, $42 \%$ reported visiting an oncologist and $87 \%$ reported visiting a PCP within the past year. Table 3 shows the results of a logistic regression modeling predictors of oncologist visits in the last 4 years. Survivors with health insurance were more likely to have visited an oncologist in the past 4 years $(\mathrm{OR}=$ $3.07, \mathrm{CI}=1.66-5.67)$. Compared with breast cancer survivors, 


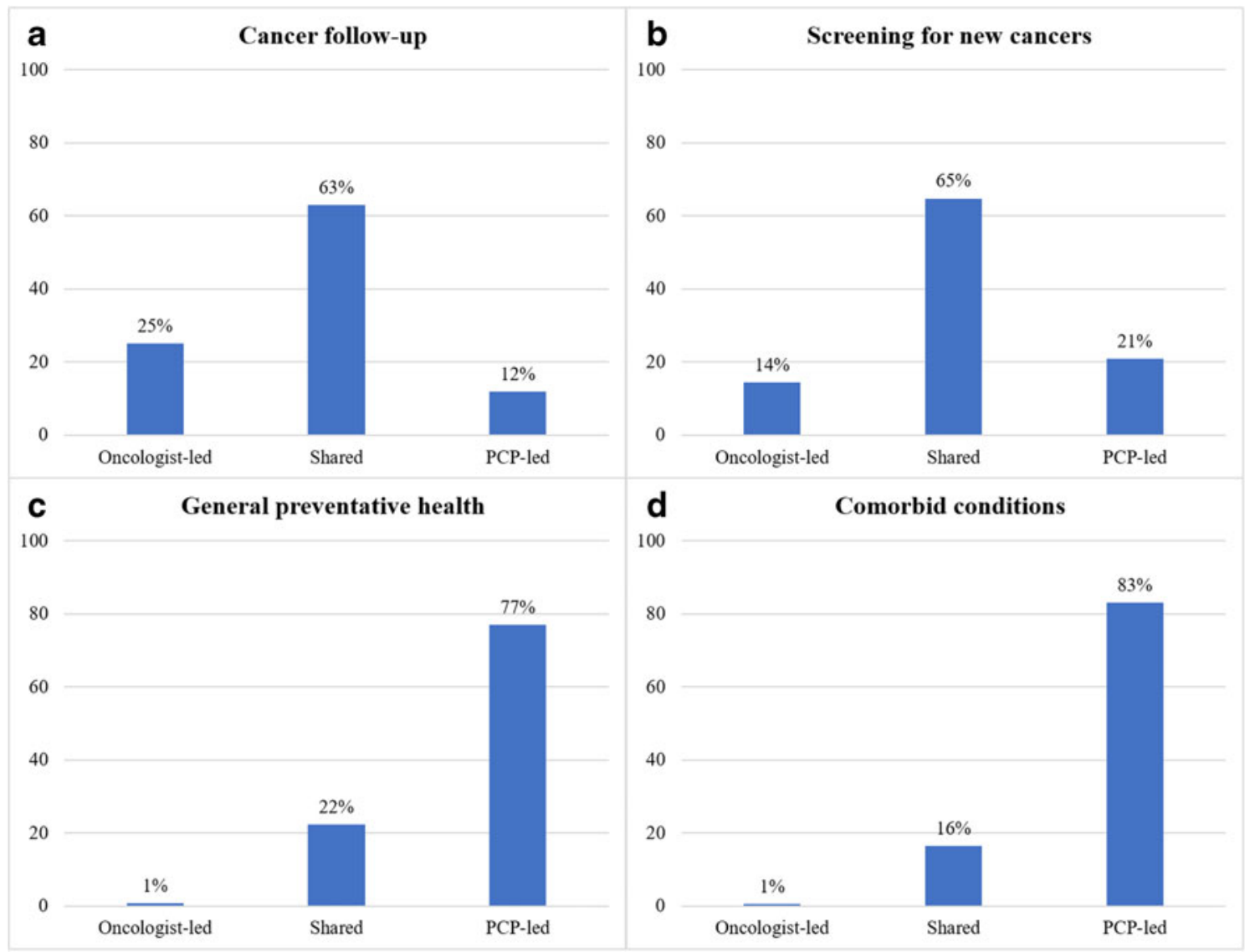

Figure 1 Long-term survivors' physician preferences for four aspects of care.

all other cancer types had significantly lower odds of seeing an oncologist in the past 4 years (see Table 3). Survivors with regional/distant cancer had greater odds of visiting an oncologist in the past 4 years $(\mathrm{OR}=2.38, \mathrm{CI}=1.89-2.99)$ compared

Table 2 Factors Associated with Preferences for Follow-up Cancer Care

\begin{tabular}{|c|c|c|c|c|c|c|}
\hline & \multicolumn{3}{|c|}{ Prefer oncologist-led to shared care } & \multicolumn{3}{|c|}{ Prefer PCP-led to shared care } \\
\hline & Odds ratio & $95 \% \mathrm{CI}$ & $P$ value & Odds ratio & $95 \% \mathrm{CI}$ & $P$ value \\
\hline Age* & 0.99 & $0.98-1.00$ & 0.06 & 1.01 & $0.99-1.02$ & 0.46 \\
\hline \multicolumn{7}{|l|}{ Race/ethnicity } \\
\hline Non-Hispanic white & Ref & & & & & \\
\hline Other & 1.08 & $0.76-1.52$ & 0.68 & 0.53 & $0.29-0.96$ & 0.04 \\
\hline \multicolumn{7}{|l|}{ Marital status } \\
\hline Married/cohabitating & Ref & & & & & \\
\hline Separated/divorced/widowed & 1.02 & $0.78-1.34$ & 0.87 & 0.97 & $0.67-1.40$ & 0.86 \\
\hline Single & 0.49 & $0.30-0.81$ & 0.01 & 0.41 & $0.18-0.91$ & 0.03 \\
\hline \multicolumn{7}{|l|}{ Education } \\
\hline High school or less & Ref & & & & & \\
\hline More than high school & 1.50 & $1.19-1.89$ & 0.0007 & 1.37 & $1.01-1.87$ & 0.05 \\
\hline \multicolumn{7}{|l|}{ Health insurance } \\
\hline Yes & 3.46 & $1.35-8.89$ & 0.01 & 0.68 & $0.31-1.53$ & 0.35 \\
\hline No & Ref & & & & & \\
\hline \multicolumn{7}{|l|}{ Cancer type by gender } \\
\hline Breast & Ref & & & & & \\
\hline Prostate & 0.69 & $0.51-0.95$ & 0.02 & 1.67 & $1.11-2.50$ & 0.01 \\
\hline Colorectal male & 0.78 & $0.49-1.27$ & 0.32 & 1.43 & $0.74-2.76$ & 0.29 \\
\hline Colorectal female & 0.66 & $0.43-1.01$ & 0.05 & 1.90 & $1.10-3.26$ & 0.02 \\
\hline Other male & 0.84 & $0.60-1.18$ & 0.32 & 1.32 & $0.80-2.18$ & 0.28 \\
\hline Other female & 0.80 & $0.61-1.05$ & 0.11 & 1.50 & $1.00-2.25$ & 0.05 \\
\hline \multicolumn{7}{|l|}{ Cancer stage } \\
\hline In situ/localized & Ref & & & & & \\
\hline Regional/distant & 1.19 & $0.95-1.48$ & 0.14 & 0.56 & $0.39-0.79$ & 0.001 \\
\hline Fear of cancer recurrence* & 0.99 & $0.98-1.01$ & 0.24 & 0.96 & $0.93-0.98$ & $<0.0001$ \\
\hline
\end{tabular}

Italicized values indicate statistical significance with a $P$ value $<0.05$

*Continuous variable 
Table 3 Factors Associated with Having Seen an Oncologist in the Last 4 Years

\begin{tabular}{llll}
\hline \hline & Odds ratio & $\mathbf{9 5 \%}$ CI & P value \\
\hline Age* & 1.00 & $0.99-1.01$ & 0.94 \\
Race/ethnicity & & & \\
Non-Hispanic white & Ref & & \\
Other & 0.80 & $0.58-1.12$ & 0.19 \\
Marital status & & & \\
Married/cohabitating & Ref & & \\
Separated/divorced/widowed & 0.87 & $0.67-1.12$ & 0.27 \\
Single & 0.86 & $0.57-1.30$ & 0.48 \\
Education & Ref & & \\
High school or less & 1.17 & $0.95-1.45$ & 0.15 \\
More than high school & & & \\
Health insurance & 3.07 & $1.66-5.67$ & 0.0003 \\
Yes & Ref & & \\
No & & & \\
Cancer type by gender & Ref & & \\
Breast & 0.19 & $0.14-0.26$ & $<0.0001$ \\
Prostate & 0.24 & $0.15-0.37$ & $<0.0001$ \\
Colorectal male & 0.30 & $0.20-0.45$ & $<0.0001$ \\
Colorectal female & 0.26 & $0.18-0.36$ & $<0.0001$ \\
Other male & 0.26 & $0.20-0.35$ & $<0.0001$ \\
Other female & & & \\
Cancer stage & Ref & & \\
In situ/localized & 2.38 & $1.89-2.99$ & $<0.0001$ \\
Regional/distant & 1.03 & $1.01-1.04$ & 0.0013 \\
Fear of cancer recurrence* & & & \\
Preferred follow-up cancer care & & & \\
Shared & Ref & $1.63-2.65$ & $<0.0001$ \\
Oncologist-led & 2.08 & $0.25-0.46$ & $<0.0001$ \\
PCP-led & 0.34 & & \\
\hline
\end{tabular}

Italicized values indicate statistical significance with a $P$ value $<0.05$ *Continuous variable

with those with localized cancer. Each added point to the fear of recurrence score significantly increased the odds of oncologist visits by $3 \%(\mathrm{CI}=1-4 \%)$. Survivors' preferences for care were predictive of actual care: those preferring oncologist-led to shared care were more likely to have seen an oncologist $(\mathrm{OR}=2.08, \mathrm{CI}=1.63-2.65)$ in the last 4 years, while those preferring PCP-led to shared care had $66 \%(\mathrm{CI}=$ $54 \%-75 \%)$ lower odds. A sensitivity analysis modeling predictors of oncology visits in the last 2 years (instead of the last 4 years) showed similar results.

\section{DISCUSSION}

Most of our population-based sample of disease-free, longterm cancer survivors preferred that PCPs and oncologists share care for their ongoing cancer follow-up (63\%) and screening (65\%). Yet only $42 \%$ had seen an oncologist in the last year, suggesting many have transitioned solely to the primary care setting. Most survivors preferred that PCPs lead their general preventive health $(77 \%)$ and comorbid condition $(83 \%)$ care. These findings suggest tangible opportunities to enhance patient-centered care for the growing population of long-term survivors.

Our study demonstrates the important role PCPs could play in long-term survivor care; however, previous research demonstrates that PCPs do not necessarily attend to survivors' cancer-related needs (e.g., surveillance for recurrence). ${ }^{13-15}$ PCP-led care of long-term survivors could be enhanced by changing policies to improve coordination of care between PCPs and oncologists, as well as providing broad-based education of PCPs in practice, CMEs, and medical school to disseminate the core competencies required for optimal survivor care. Resources in these areas are emerging but more are needed. ${ }^{33}$ Guidelines designed for PCPs and providing comprehensive coverage of survivorship issues - surveillance for recurrence, screening for new primary cancers, assessment and management of physical and psychosocial long-term and late effects of cancer and its treatment, health promotion, and care coordination and practice implications - are available for four common cancers: colorectal, breast, prostate, and head and neck. ${ }^{34-37}$

We found a potential disconnect between survivor preferences and actual care: despite half of survivors not seeing an oncologist recently, $88 \%$ wanted an oncologist involved in their follow-up cancer care (63\% shared, $25 \%$ oncologist-led). This discrepancy suggests some survivors are not receiving preferred care, which could lower survivor satisfaction and adherence to provider recommendations. ${ }^{27,}{ }^{38}$ Increasing oncologist visits is unlikely, given oncologists' current workload and expected future shortages. ${ }^{39}$ Further, research among breast cancer survivors suggests that recurrence-related events and quality of life are similar for those followed by family physicians or specialists, ${ }^{40}$ and specialist-led follow-up care for these patients is associated with higher costs. ${ }^{41}$ A US study of older patients with multiple morbidities, including cancer, showed higher costs, lower continuity of care, and similar outcomes for those seen by specialists as opposed to PCPs. ${ }^{18}$ Comorbidities are highly prevalent among survivors leading to poorer survival, lower quality of life, and higher costs. ${ }^{42,43}$ Given the existing literature, oncologist involvement in survivor care via traditional office visits may not be in survivors' or society's best interests.

Two approaches could address the conflict of survivors preferring oncologist involvement when oncologist office visits are not practical. The first approach could involve creative, low-resource strategies to maintain some oncologist involvement such as e-Consults or agreements regarding followup care. This approach aligns with the patient-centered medical home (PCMH) which is intended to provide comprehensive care by a well-coordinated team of clinicians, including specialists, and led by a personal physician who is often a PCP. $^{26,44}$ The PCMH may match well with survivors' varied needs. ${ }^{45,}{ }^{46}$ While evolving evidence suggests the PCMH can improve patient and clinician experiences, decrease emergency room visits, and improve adherence and clinical outcomes, it comes mostly from non-cancer samples. ${ }^{47,}, 48$

The second approach involves influencing survivors' preferences. When patient preferences cannot be reasonably honored, the principles of patient-centered care suggest that clinicians may help patients reach a new understanding of how best to achieve their health goals. ${ }^{26}$ Several strategies could sway the opinions of long-term survivors preferring but not requiring oncologist care. Early in survivorship, oncologists should 
discuss the plans to transfer survivors into PCP care with explicit reassurances that the patients may return to oncology if needed. Survivor and oncologist concerns that PCPs are unwilling or have insufficient time or expertise to conduct cancer follow-up ${ }^{10,49,50}$ might be addressed by conversations about care responsibilities, identifying PCPs with additional training or interest in cancer survivorship, ${ }^{33}$ and reinforcement that research suggests similar outcomes for survivors seen by PCPs versus oncologists. ${ }^{40}$

We found several factors related to care preferences and oncologist visits. First, higher levels of fear of recurrence predicting preference for oncologist care and oncologist visits suggest an avenue of intervention. Practical, evidence-based information on how to discuss, assess, and manage fear of recurrence are provided in a recently published guide. ${ }^{29}$ Primary care clinician knowledge and self-efficacy for identifying and addressing fear of recurrence can be improved through education. ${ }^{51}$ Second, in our models, health insurance was associated with increased survivor preference for oncologistled cancer follow-up care and actual oncologist visits, likely reflecting fewer financial barriers among the insured. Third, survivors with regional/distant stage were also more likely to both prefer and receive oncologist care, which aligns with proposals to risk stratify survivor care based on disease severity (e.g., stage) to identify the optimal model of care. ${ }^{33,52}$ Finally, survivors preferring oncologist-led care were more likely to see an oncologist, suggesting preference influences care decisions.

The strengths of this paper include a large, population-based sample of long-term survivors of ten cancers whose diagnosis is confirmed by cancer registries. As with most survey research, the results of this study may be subject to bias due to missing information, inaccurate recall, or other issues. Our measurement instruments only accounted for physician-centric care, but other clinicians (e.g., nurse practitioner, physician assistant, case manager, navigator) may be increasingly important given expected shortages of $\mathrm{PCPs}^{53}$ and oncologists ${ }^{39}$.

Our paper provides insights into long-term survivors' preferences for and use of PCPs and oncologists. To assure patient-centered care, clinicians should assess patient preferences, understand the factors that influence preferences, and offer care that considers preferences along with risk factors for recurrence, late effects, and comorbidities. ${ }^{33,52}$ Survivors in our sample wanted PCPs to lead or collaborate on the four types of care addressed in our study. This finding aligns with models of care that designate the PCP as the lead of a wellcoordinated team of clinicians which includes specialists (e.g., PCMH, medical neighborhood). ${ }^{26,44,54}$ This approach enables the use of oncologists' strengths when needed and may address survivor preferences for their continued involvement in cancer follow-up care. When oncologist visits are not practical or warranted, efforts may be made to influence survivor preferences by showing them alternate pathways to meet their health goals. As most long-term survivors receive care from PCPs, there is a continuing need to develop and disseminate survivorship care educational resources designed specifically for PCPs as well as systems and policies aimed to provide comprehensive, coordinated care for cancer survivors.

ACKNOWLEDGMENTS: Certain data used in this study were obtained from the Connecticut Tumor Registry located in the Connecticut Department of Public Health. Name, phone number, address, doctor name, social security number, date of birth, date of diagnosis, gender, cancer type, primary site, race, Hispanic origin, and stage and grade at diagnosis study were obtained from the Connecticut Tumor Registry located in the Connecticut Department of Public Health. The authors thank all the study participants; the physicians of the participants; Susan Higgins, MD; Project Director Annie O'Neill; Rajni Mehta, Director of the Rapid Case Ascertainment Shared Resource of the Yale Cancer Center and the following Connecticut Hospitals: Charlotte Hungerford Hospital, Bridgeport Hospital, Danbury Hospital, Hartford Hospital, Middlesex Hospital, New Britain General Hospital, Bradley Memorial Hospital, Yale/New Haven Hospital, St. Francis Hospital and Medical Center, St. Mary's Hospital, Hospital of St. Raphael, St. Vincent's Medical Center, Stamford Hospital, William W. Backus Hospital, Windham Hospital, Eastern Connecticut Health Network, Griffin Hospital, Bristol Hospital, Johnson Memorial Hospital, Day Kimball Hospital, Greenwich Hospital, Lawrence and Memorial Hospital, Milford Hospital, New Milford Hospital, Norwalk Hospital, Sharon Hospital, and Waterbury Hospital.

Corresponding Author: Tenbroeck G. Smith, MA; Behavioral and Epidemiology Research Group, American Cancer Society, 250 Williams Street, Atlanta, GA 30303, USA (e-mail: tenbsmith@yahoo.com).

Funders The American Cancer Society (ACS) Studies of Cancer Survivors (SCS) were funded by the Intramural Research Department of the ACS.

\section{COMPLIANCE WITH ETHICAL STANDARDS:}

The authors assume full responsibility for analyses and interpretation of the data collected from these data, which do not necessarily reflect the views of the American Cancer Society.

Conflict of Interest: The authors declare that they do not have a conflict of interest.

\section{REFERENCES}

1. Miller KD, Siegel RL, Lin CC, et al. Cancer treatment and survivorship statistics, 2016. CA Cancer J Clin. 2016;66(4):271-289.

2. Cancer Treatment \& Survivorship Facts \& Figures 2016-2017. Atlanta: American Cancer Society;2016.

3. Mayer DK, Nasso SF, Earp JA. Defining cancer survivors, their needs, and perspectives on survivorship health care in the USA. Lancet Oncol. 2017;18(1):e11-e18.

4. Mariotto AB, Rowland JH, Ries LA, Scoppa S, Feuer EJ. Multiple cancer prevalence: a growing challenge in long-term survivorship. Cancer Epidemiol Biomarkers Prev. 2007;16(3):566-571.

5. Curigliano G, Cardinale D, Dent S, et al. Cardiotoxicity of anticancer treatments: Epidemiology, detection, and management. CA Cancer J Clin. 2016;66(4):309-325.

6. Kline RM, Arora NK, Bradley CJ, et al. Long-Term Survivorship Care After Cancer Treatment - Summary of a 2017 National Cancer Policy Forum Workshop. Journal of the National Cancer Institute. 2018;110(12): 1300-1310.

7. Shapiro CL, Jacobsen PB, Henderson T, et al. ReCAP: ASCO Core Curriculum for Cancer Survivorship Education. Journal of oncology practice. 2016;12(2):145, e108-117.

8. Cheung WY, Neville BA, Cameron DB, Cook EF, Earle CC. Comparisons of patient and physician expectations for cancer survivorship care. $J$ Clin Oncol. 2009;27(15):2489-2495. 
9. Dossett LA, Hudson JN, Morris AM, et al. The primary care provider (PCP)-cancer specialist relationship: A systematic review and mixedmethods meta-synthesis. CA Cancer J Clin. 2017;67(2):156-169.

10. Potosky AL, Han PK, Rowland J, et al. Differences between primary care physicians' and oncologists' knowledge, attitudes and practices regarding the care of cancer survivors. J Gen Intern Med. 2011;26(12):1403-1410.

11. Cheung WY, Aziz N, Noone AM, et al. Physician preferences and attitudes regarding different models of cancer survivorship care: a comparison of primary care providers and oncologists. Journal of cancer survivorship : research and practice. 2013;7(3):343-354.

12. Nekhlyudov L, Aziz NM, Lerro C, Virgo KS. Oncologists' and primary care physicians' awareness of late and long-term effects of chemotherapy: implications for care of the growing population of survivors. Journal of oncology practice. 2014;10(2):e29-36.

13. Field TS, Doubeni C, Fox MP, et al. Under utilization of surveillance mammography among older breast cancer survivors. $J$ Gen Intern Med. 2008;23(2):158-163.

14. Keating NL, Landrum MB, Guadagnoli E, Winer EP, Ayanian JZ. Factors related to underuse of surveillance mammography among breast cancer survivors. J Clin Oncol. 2006;24(1):85-94.

15. McBean AM, Yu X, Virnig BA. The use of preventive health services among elderly uterine cancer survivors. Am J Obstet Gynecol. 2008; 198(1):86.e81-88

16. Klabunde CN, Han PK, Earle CC, et al. Physician roles in the cancerrelated follow-up care of cancer survivors. Fam Med. 2013;45(7):463-474.

17. Snyder CF, Frick KD, Peairs KS, et al. Comparing care for breast cancer survivors to non-cancer controls: a five-year longitudinal study. $J$ Gen Intern Med. 2009;24(4):469-474.

18. Bynum JPW, Chang CH, Austin A, Carmichael D, Meara E. Outcomes in Older Adults with Multimorbidity Associated with Predominant Provider of Care Specialty. J Am Geriatr Soc. 2017;65(9): 1916-1923.

19. Snyder CF, Frick KD, Herbert RJ, et al. Comorbid condition care quality in cancer survivors: role of primary care and specialty providers and care coordination. Journal of cancer survivorship : research and practice. 2015;9(4):641-649.

20. NCCN clinical practice guidelines: Survivorship (Version 1.2019). https:// www.nccn.org/professionals/physician_gls/pdf/survivorship.pdf. Accessed May 22, 2019

21. Halpern MT, Viswanathan M, Evans TS, Birken SA, Basch E, Mayer DK. Models of Cancer Survivorship Care: Overview and Summary of Current Evidence. Journal of oncology practice. 2015;11(1):e19-27.

22. Baxi SS, Kale M, Keyhani S, et al. Overuse of Health Care Services in the Management of Cancer: A Systematic Review. Medical care. 2017;55(7):723-733.

23. Carpentier MY, Vernon SW, Bartholomew LK, Murphy CC, Bluethmann SM. Receipt of recommended surveillance among colorectal cancer survivors: a systematic review. Journal of cancer survivorship : research and practice. 2013;7(3):464-483.

24. Han PK, Klabunde CN, Noone AM, et al. Physicians' beliefs about breast cancer surveillance testing are consistent with test overuse. Medical care. 2013;51(4):315-323.

25. Committee on Quality Health Care in America, Institute of Medicine. Crossing the quality chasm : a new health system for the 21st century. Washington, D.C.: National Academy Press; 2001.

26. Braddock CH, 3rd, Snyder L, Neubauer RL, Fischer GS. The patientcentered medical home: an ethical analysis of principles and practice. $J$ Gen Intern Med. 2013;28(1):141-146.

27. Rathert C, Wyrwich MD, Boren SA. Patient-centered care and outcomes: a systematic review of the literature. Med Care Res Rev. 2013;70(4):351-379.

28. Smith T, Stein KD, Mehta $\mathbf{C C}$, et al. The rationale, design, and implementation of the American Cancer Society's studies of cancer survivors. Cancer. 2007;109(1):1-12.

29. Butow P, Sharpe L, Thewes B, Turner J, Gilchrist J, Beith J. Fear of Cancer Recurrence: A Practical Guide for Clinicians. Oncology. 2018;32(1):32-38.

30. Simard S, Thewes B, Humphris G, et al. Fear of cancer recurrence in adult cancer survivors: a systematic review of quantitative studies. Journal of cancer survivorship : research and practice. 2013;7(3):300-322.

31. Simard S, Savard J. Fear of Cancer Recurrence Inventory: development and initial validation of a multidimensional measure of fear of cancer recurrence. Support Care Cancer. 2009;17(3):241-251.

32. Simard S, Savard J. Screening and comorbidity of clinical levels of fear of cancer recurrence. Journal of cancer survivorship : research and practice. 2015;9(3):481-491.
33. Nekhlyudov L, O'Malley D M, Hudson SV. Integrating primary care providers in the care of cancer survivors: gaps in evidence and future opportunities. Lancet Oncol. 2017;18(1):e30-e38.

34. Cohen EE, LaMonte SJ, Erb NL, et al. American Cancer Society Head and Neck Cancer Survivorship Care Guideline. CA Cancer J Clin. 2016;66(3):203-239.

35. El-Shami $\mathbf{K}$, Oeffinger $\mathbf{K C}$, Erb NL, et al. American Cancer Society Colorectal Cancer Survivorship Care Guidelines. CA Cancer J Clin. 2015;65(6):428-455.

36. Runowicz CD, Leach CR, Henry NL, et al. American Cancer Society/ American Society of Clinical Oncology Breast Cancer Survivorship Care Guideline. CA Cancer J Clin. 2016;66(1):43-73.

37. Skolarus TA, Wolf AM, Erb NL, et al. American Cancer Society prostate cancer survivorship care guidelines. CA Cancer J Clin. 2014;64(4):225-249.

38. Martin LR, Williams SL, Haskard KB, Dimatteo MR. The challenge of patient adherence. Ther Clin Risk Manag. 2005; 1(3): 189-199.

39. Yang W, Williams JH, Hogan PF, et al. Projected supply of and demand for oncologists and radiation oncologists through 2025: an aging, betterinsured population will result in shortage. Journal of oncology practice. 2014;10(1):39-45.

40. Grunfeld E, Levine MN, Julian JA, et al. Randomized trial of long-term follow-up for early-stage breast cancer: a comparison of family physician versus specialist care. J Clin Oncol. 2006;24(6):848-855.

41. Grunfeld E, Gray A, Mant D, et al. Follow-up of breast cancer in primary care vs specialist care: results of an economic evaluation. $\mathrm{Br}$ J Cancer. 1999;79(7-8):1227-1233.

42. Sarfati D, Koczwara B, Jackson C. The impact of comorbidity on cancer and its treatment. CA Cancer J Clin. 2016;66(4):337-350.

43. Sogaard M, Thomsen RW, Bossen KS, Sorensen HT, Norgaard M. The impact of comorbidity on cancer survival: a review. Clin Epidemiol. 2013;5(Suppl 1):3-29.

44. Agency for Healthcare Research and Quality. Defining the PCMH. https://pcmh.ahrq.gov/page/defining-pcmh. Accessed May 22, 2019.

45. Hudson Sv, Miller SM, Hemler J, et al. Adult cancer survivors discuss follow-up in primary care: 'not what i want, but maybe what i need'. Ann Fam Med. 2012;10(5):418-427.

46. Nekhlyudov L, Levit L, Hurria A, Ganz PA. Patient-centered, evidencebased, and cost-conscious cancer care across the continuum: Translating the Institute of Medicine report into clinical practice. CA Cancer $J$ Clin. 2014;64(6):408-421

47. Jackson GL, Powers BJ, Chatterjee R, et al. Improving patient care. The patient centered medical home. A Systematic Review. Ann Intern Med. 2013;158(3):169-178.

48. van den Berk-Clark C, Doucette E, Rottnek F, et al. Do PatientCentered Medical Homes Improve Health Behaviors, Outcomes, and Experiences of Low-Income Patients? A Systematic Review and MetaAnalysis. Health Serv Res. 2018;53(3):1777-1798.

49. Lewis RA, Neal RD, Hendry $\mathbf{M}$, et al. Patients' and healthcare professionals' views of cancer follow-up: systematic review. $\mathrm{Br} \mathrm{J} \mathrm{Gen}$ Pract. 2009;59(564):e248-259.

50. Neuman HB, Jacobs EA, Steffens NM, et al. Oncologists' perceived barriers to an expanded role for primary care in breast cancer survivorship care. Cancer medicine. 2016;5(9):2198-2204.

51. Berrett-Abebe J, Cadet T, Nekhlyudov L, Vitello J, Maramaldi P. Impact of an Interprofessional Primary Care Training on Fear of Cancer Recurrence on Clinicians' Knowledge, Self-Efficacy, Anticipated Practice Behaviors, and Attitudes Toward Survivorship Care. J Cancer Educ. 2018.

52. McCabe MS, Partridge AH, Grunfeld E, Hudson MM. Risk-based health care, the cancer survivor, the oncologist, and the primary care physician. Semin Oncol. 2013;40(6):804-812.

53. Projecting the Supply and Demand for Primary Care Practitioners Through 2020. Rockville, Maryland: U.S. Department of Health and Human Services;2013

54. Pham HH. Good neighbors: how will the patient-centered medical home relate to the rest of the health-care delivery system? $J$ Gen Intern Med. 2010;25(6):630-634.

Publisher's Note Springer Nature remains neutral with regard to jurisdictional claims in published maps and institutional affiliations. 